\title{
The potential of health literacy to address the health related UN sustainable development goal 3 (SDG3) in Nepal: a rapid review
}

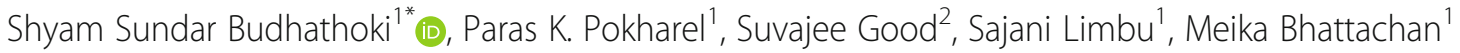
and Richard H. Osborne ${ }^{3}$

\begin{abstract}
Background: Health literacy has been linked to health outcomes across population groups around the world. Nepal, a low income country, experiences the double burden of highly prevalent communicable as well as non-communicable diseases. The World Health Organization (WHO) has positioned health literacy as a key mechanism to meet the health-related Sustainable Development Goal (SDG3). However, there is little known about the status of health literacy in developing countries such as Nepal. This paper aims to review the potential of health literacy to address SDG3 in Nepal.

Methods: A rapid review was conducted using the knowledge to action evidence summary approach. Articles included in the review were those reporting on barriers to health care engagements in Nepal published in English language between January 2000 and December 2015.

Results: Barriers for healthcare engagement included knowledge and education as strong factors, followed by culture, gender roles, quality of service and cost of services. These barriers influence the Nepalese community to access and engage with services, and make and enact healthcare decisions, not only at the individual level but at the family level. These factors are directly linked to health literacy. Health literacy is a pivotal determinant of understanding, accessing and using health information and health services, it is important that the health literacy needs of the people be addressed.
\end{abstract}

Conclusion: Locally identified and developed health literacy interventions may provide opportunities for systematic improvements in health to address impediments to healthcare in Nepal. Further research on health literacy and implementation of health literacy interventions may help reduce inequalities and increase the responsiveness of health systems which could potentially facilitate Nepal to meet the sustainable development goals. While there is currently little in place for health literacy to impact on the SDG3, this paper generates insights into health literacy's potential role.

Keywords: Health literacy, Health system responsiveness, Impediments to public health in Nepal, Development, Nepal, Sustainable development goals, SDG, Equity

\footnotetext{
* Correspondence: ss.budhathoki@gmail.com

'School of Public Health \& Community Medicine, B P Koirala Institute of

Health Sciences, Dharan 56700, Nepal

Full list of author information is available at the end of the article
} 


\section{Background}

Health Literacy is defined as "the cognitive and social skills which determine the motivation and ability of individuals to gain access to, understand and use information in ways which promote and maintain good health" [1]. It incorporates the characteristics of an individual along with the supports needed to access, understand, appraise and use the information and services to make decisions about their health and the health of their family and the community [2].

Low health literacy is associated with inadequate knowledge about the health as well as the healthcare system, poor access and utilisation of health services and also increased hospitalization. This leads to poor health outcomes and health inequalities [3-6]. Dimensions of health literacy include cognitive, affective, social and personal skills and attributes [7, 8]. A comprehensive understanding of health literacy is essential to understand the full range of needs of members of the community in order to provide accessible and equitable services to all [2]. Furthermore, having an understanding of the health literacy needs of individuals and communities provides the opportunity to develop interventions to improve health outcomes and reduce inequalities $[9,10]$.

\section{Nepal- a low income country with substantial health and development challenges}

Nepal is a low income country, ranking seventh among the eight South Asian countries and 147 of 187 countries in the world [11]. The life expectancy at birth in Nepal is 68 years [12]. The country's population is 26.4 million, with $83 \%$ living in rural areas [13, 14]. One fourth of the population lives below the poverty line [15] and the adult literacy rate is $66 \%$ however the literacy rate in females is lower at $57 \%$ [13].

The doctor to population ratio in Nepal is $0.37 / 1,000$ people (as low as 0.008 in rural areas and 1.5/1,000 people in the capital city). Individuals bear $55 \%$ of total healthcare expenditure as out-of-pocket payments [16]. About two thirds of healthcare in the acute sector is provided by private hospitals [17]. Gaps to address the social determinants of health exist in Nepal. While Nepal still faces a burden of infectious diseases struggling with inadequate basic hygiene and sanitation along with deep rooted cultural beliefs, the burden of noncommunicable diseases is also on the rise $[18,19]$. Limited research has been found mentioning health literacy in Nepal [20-22] and level of health literacy of the people of Nepal is not known.

\section{Health-related sustainable development Goal 3 (SDG3)}

While SDG3 Ensure healthy lives and to promote wellbeing at all ages is the only specific health goal among the SDGs, other goals, e.g., SDG1 (No poverty), SDG2 (Zero hunger),
SDG4 (Quality education), SDG8 (Decent work and economic growth), and SDG10 (Reduced inequalities) are linked to health and will contribute to improvement of overall population health. SDG3 addresses maternal health, neonatal and child health, AIDS, tuberculosis, malaria and includes universal access to sexual and reproductive health services including family planning. Nepal made progress with the Millennium Development Goals through improvements in maternal and child health. With these achievements, Nepal, like all other countries, is to now set to work towards achieving the SDGs by 2030.

At the 9th Global Conference on Health Promotion 2016, the World Health Organization (WHO) launched the Shanghai Declaration where health literacy is positioned as a foundation block for health and sustainable development in the coming decades [23]. However previous research in Nepal has paid little attention to health literacy, that is, how people and the community might be empowered to engage in recognizing health needs, how to improve knowledge about the health system, and enabling people to regard access to health services as a right. These individual and community attributes are critical components of health literacy and it is critical to understand these such that health literacy can be used to assist with overcoming such impediments and strengthen the health system, improve health outcomes, and, ultimately, to meet the SDG3 in Nepal. This paper discusses the potential of health literacy to address the known and potential impediments for health in Nepal to meet the health-related SDG3.

\section{Methods}

Study design

A rapid review was conducted using the knowledge to action evidence summary approach [24]. The review question was: What are the impediments of public health in Nepal that could potentially be addressed by health literacy?

\section{Search strategy}

The key words used were taken from the WHO SEARO Health Literacy Toolkit [2] as: 'access', 'appraise', 'understand', 'decide', 'availability', 'accessibility', 'healthcare', 'utilisation', 'health service', 'ability to decide', 'decision making in health', 'willingness to engage', 'health system responsiveness' in different combinations with 'impediments' and 'barriers'; with 'health' and 'Nepal'. We limited our search engines to Pubmed, Google Scholar and Nepal Journal Online.

\section{Inclusion and exclusion criteria}

The inclusion criteria were set to include all articles published between January 2000 and December 2015 and reporting on factors influencing healthcare seeking 
and utilization in Nepal. Articles not published in English were excluded. All articles identified in the search were subjected to the filtering process as shown in Fig. 1.

\section{Theoretical framework}

While many barriers were identified that influenced geographical accessibility to healthcare [25], we used a conceptual framework to categorize barriers for engagement in healthcare (income and price, culture and gender, knowledge and education, and quality of services) as adapted from the access to healthcare in developing countries model by O’Donnel [26].

\section{Data extraction}

Two reviewers independently performed title, abstract and content analysis for the matching the inclusion criteria. A data extraction form was used to record the factors influencing healthcare and disagreement between the two researchers were agreed upon through consensus by the whole research team.

\section{Synthesis of review}

The identified factors were then classified using four categories; income and price, culture and gender, knowledge and education, and quality of services. To further organize the literature to reveal potential levels of health literacy action we used the four causal paths described by Batterham et al. (Fig. 2) [9].

\section{Quality assessment}

The Assessment, Development and Evaluation (GRADE) approach [27] was used to assess the quality of evidence for trials, case control, cross-sectional and qualitative studies and the AMSTAR checklist was used to assess the quality of the reviews [28]. The articles extracted were independently assessed for quality by two reviewers and if a disagreement occurred a third team member undertook an addition review and negotiated a consensus.

\section{Results}

Overall, 38 original articles included in the review covered a variety of factors influencing healthcare service utilisation (Table 1). There are 5 review articles, 1 trial, 1 case control, 24 cross-sectional and 7 qualitative studies included in this review. The review articles included are of either medium or low quality as classified by the AMSTAR checklist. The trial was of medium quality and the observational studies were either low quality or very low quality as classified using the GRADE approach.

\section{Income and price}

Six studies indicated that cost of services is an important barrier for health service utilisation in Nepal. Low annual income, unemployment and inability to bear travel costs to reach health facilities for treatment were associated with less utilisation of tuberculosis treatment [29]. Household income played a role in illness reporting and subsequent healthcare seeking [30-33]. For children in poorer households, healthcare seeking was postponed until urgent [34].

\section{Culture and gender}

Eleven studies reported on cultural practice and perceived gender roles that influence health seeking behaviour. Gender roles affects illness reporting, healthcare decision making and health expenditure [31]. Healthcare seeking was considered as an investment in the family, however male children were more likely to receive care earlier [31, 35]. Fewer women were involved in household decision making processes. Women who participated in household decision making and those who discussed

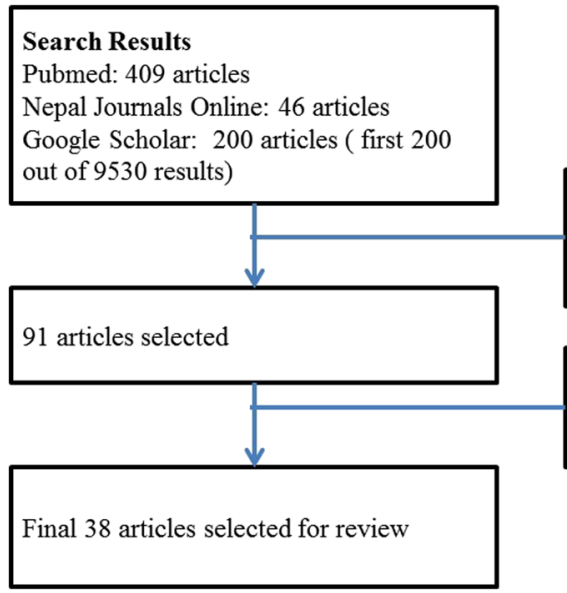

564 articles excluded (removal of duplicates, not fitting the inclusion criteria)

53 articles excluded after reading full text (not relevant to research question)

Fig. 1 Article selection process 


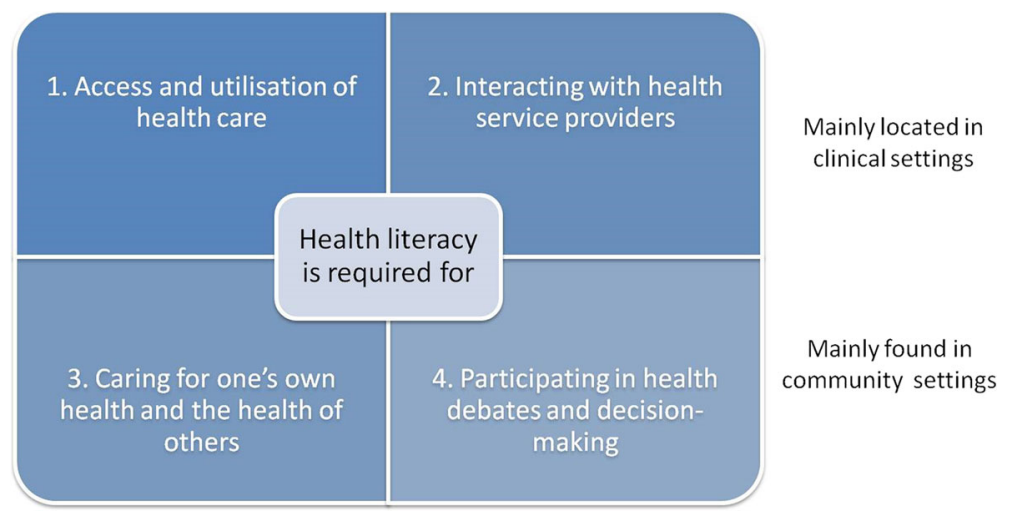

Fig. 2 Causal pathway for health literacy influencing health outcome [9]

health issues with their husbands were more likely to use maternal healthcare services [36-39]. Women who received health education along with their husbands were more likely to take care of their own health [40].

Women with higher level of autonomy could negotiate safe sex [41]. Malnutrition was lower in children where their mother had more decision making power [42]. Husbands influenced decisions about care seeking [38, 43-45]. Gender discrimination was seen to increase vulnerability of migrant women for sexually transmitted infections and HIV [46]. Seeking healthcare from traditional healers was common in mountainous regions. The perception of high cost of hospital services was seen as a reason for consulting traditional healers [47].

\section{Knowledge and education}

Among 18 identified studies, pregnant women were less aware of free birthing services [48] including support for transportation to health institutions [49]. There was low awareness of disease as well as the risk of health-related economic burden to the family [50]. Women with higher education were more likely to seek healthcare [32, 33, 36, 38, 51, 52]. Seeking healthcare was less frequent among illiterate women [53]. Educated husbands were more likely to facilitate their wives to visit health facilities [30, 51]. Family planning uptake including the choice of family planning by the women was associated with the husband's education [54]. Women often had limited understanding of early danger signs and the ways to avoid pregnancy complications [55]. Women who were able to recognise the warning signs of pregnancy complications were more likely to utilise skilled birth attendants (SBA) during deliveries [56]. Increasing awareness among women appeared to increase the uptake of SBA services [57].

Inadequate access to information, as well as services, is a major barrier for young people in the uptake of sexual and reproductive health services $[58,59]$. A knowledge to practice gap had been reported in some occupational groups; educated welders were more aware of hazards and more likely to use personal protective equipment [60], and migrant workers, who lack knowledge of diseases were more likely to be engaged in unsafe sex and be exposed to HIV [61], mostly due to low perceived vulnerability [62].

\section{Quality of services}

Among 7 studies, impediments for effective health service delivery were found to be due to poor infrastructure, lack of services, poor communication between health workers and patients, staff shortages and attitudes of clinicians at health institutions that hinder the uptake of services [53, 63]. Low competency of managers to implement programs, delays in disbursement of funds, lack of policy communication among providers and public resulted in suboptimal performance of health programs [49]. Barriers to utilisation of health services were lack of confidentiality, negative attitudes of the healthcare providers and inadequate communication between providers and the patients [64]. Dissatisfaction from service providers' attitudes and practices lead to under-utilisation of services in a mountainous region [65]. Availability of comprehensive health services was associated with higher utilisation of healthcare [66]. Perceived better quality services in private institutions drove people away from public institutions towards private healthcare institutions [47].

\section{Discussion}

This review has provided an understanding of factors affecting the healthcare engagement by the people of Nepal. These factors are in line with the WHO list of social determinants of health $(\mathrm{SDH})$ [67] that exist as impediments to attain the SDGs. While the SDG3 requires multi-sectoral approach beyond the health sector, addressing the social determinants of health and attaining universal health coverage are essential routes 


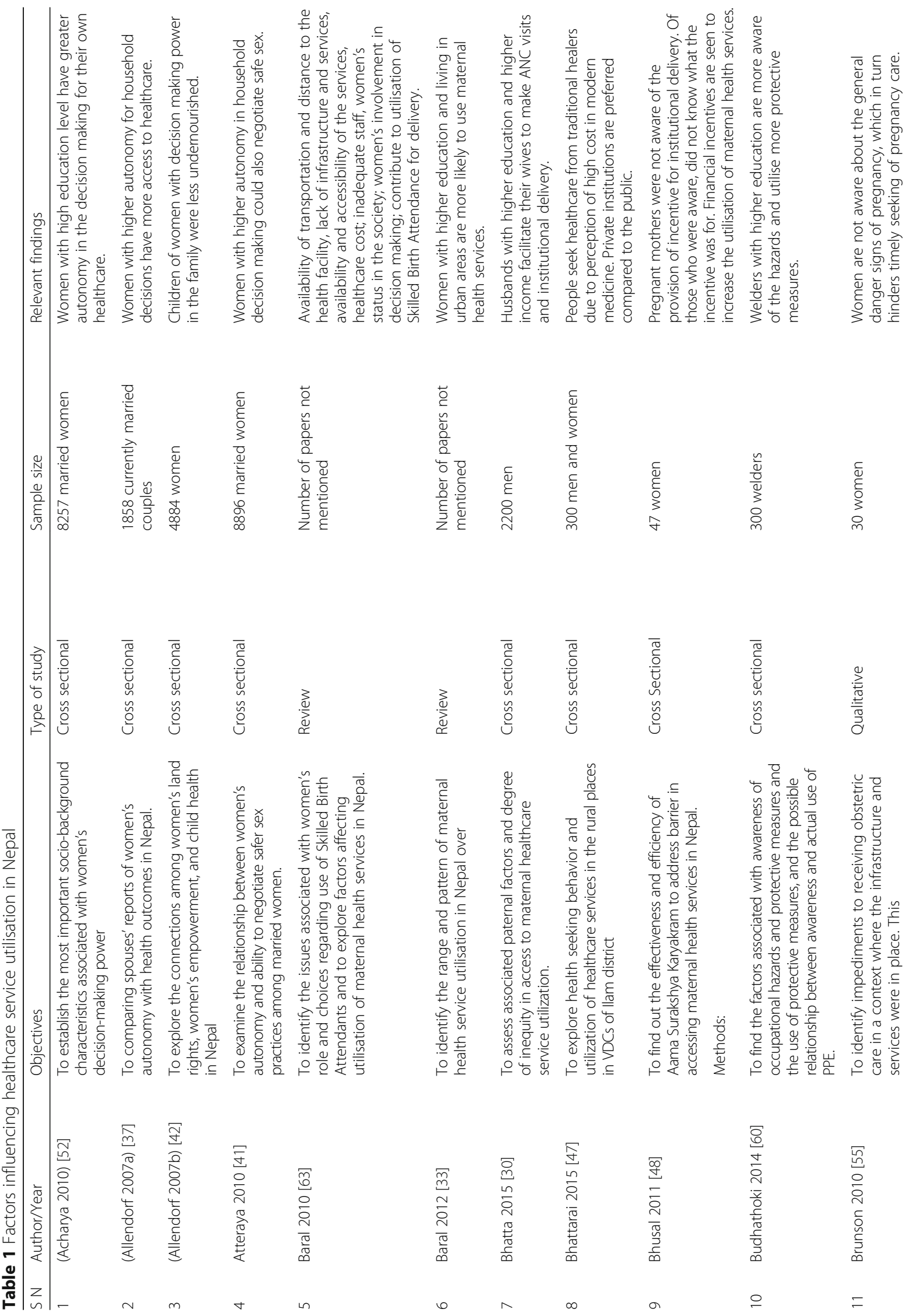




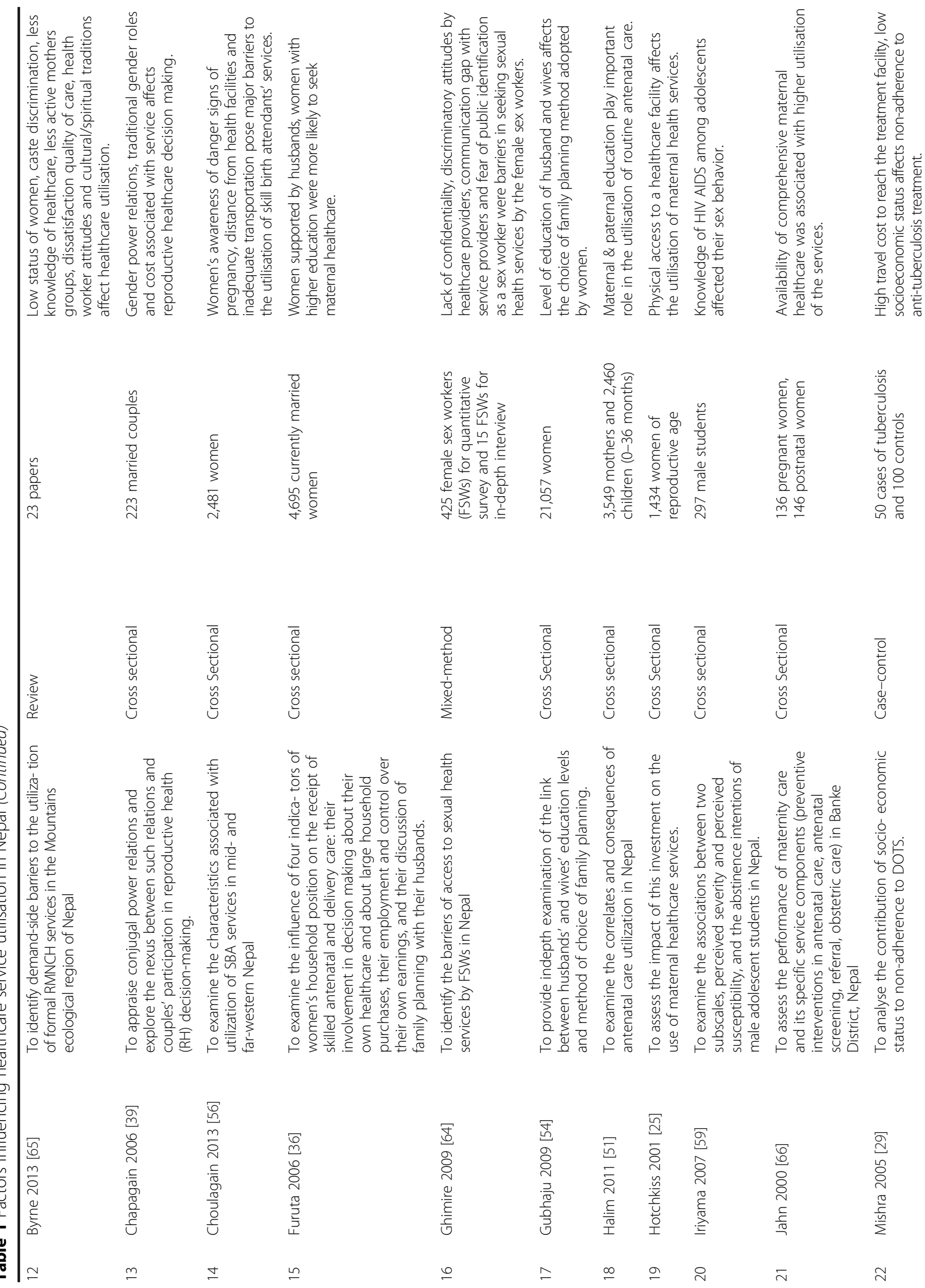




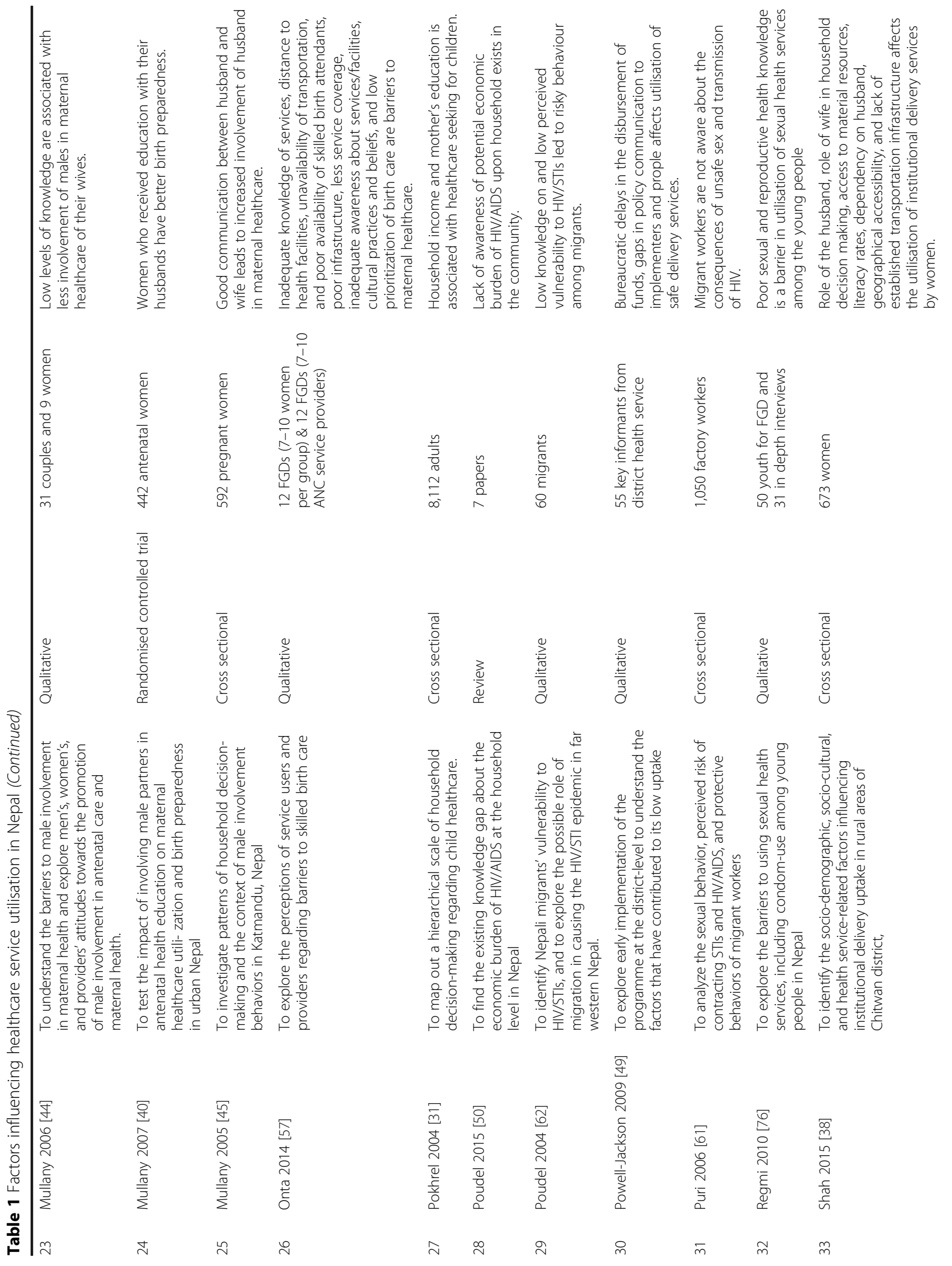




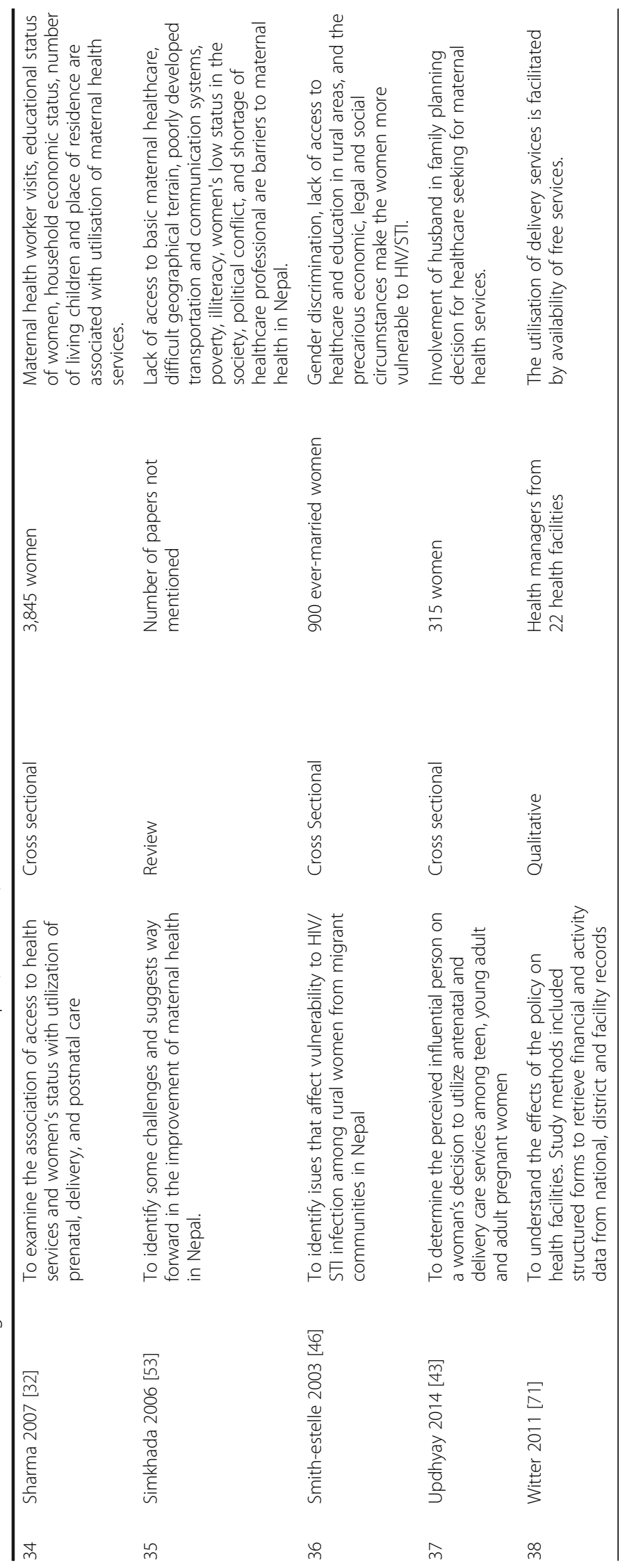


to the attainment of SDG3 [68]. Overall, the most consistent and strongest factor influencing health services utilisation in this review appears to be knowledge and education. Culture and gender roles are also important for Nepal, being a country with 125 ethnic groups and 123 spoken languages [13], with clear evidence of gender inequality which is embedded in local cultures, being linked to health inequality. Measuring health literacy and designing health literacy interventions provides system level solutions to address self-care, disease management and improve system responsiveness in different population groups $[9,69,70]$. Among the identified impediments to public health in this review, health literacy could address social determinants of health that are related to knowledge, education, communication, culture and gender roles and quality of service by empowering people to take care of themselves, families and communities [2].

While income and price factors are likely to be addressed, in part, through universal health coverage, work needs to be done to ensure the population is aware of the services, and that they are free [71]. Public health interventions in Nepal will need to include a focus on improving education, including health education, gender equity with careful consideration of cultural diversity, and strengthening the health system. While literacy of the population is linked with health, research linking health literacy with health outcomes has not yet been undertaken in Nepal. The potential pathways [9] for health literacy to impact on health and equity are different for clinical settings and community settings. Health literacy determinants for factors related to access and communication with healthcare providers are more relevant for clinical settings and the factors related to caring and decision making are more relevant for community settings. See Table 2 for a summary of potential causal pathways for impacting on health and equity in Nepal.

\section{Relationship between factors influencing public health, SDGs and health literacy}

In this review we only focused on health-related SDG3. However, there are clear links between health literacy and SDG1 (No poverty), SDG2 (Zero hunger), SDG4 (Quality education), SDG8 (Decent work and economic growth), SDG9 (Industry, information and infrastructure), SDG10 (Reduced inequalities) and SDG16 (Peace, justice and strong institutions) [23]. The areas for health literacy interventions identified are likely to have impact on these as they are in line with the causal pathways identified by Batterham et al. [9]. Framing the interventions in this way is useful because it identifies starting points for programmatic interventions. In health service settings the focus may be more on the health literacy strengths and limitations of individuals seeking care, the levels of engagement they are able to have with the services, and the ways in
Table 2 Summary of potential health literacy-related causal pathways for impacting on health and equity in Nepal

1. Health literacy is required to enable people to access and utilize healthcare

People in Nepal have many potential barriers to access and use healthcare services. Barriers include cost of services, cost of transport, low income and unemployment. Existing gender roles and discrimination related to local culture, knowledge of services and health problems, limited availability of services, low quality services provide large challenges for people to access and utilize services.

To overcome these barriers, the health literacy of community members needs to be high such that people are empowered to be able to make decisions about healthcare and overcome access barriers.

2. Health literacy is required to enable people to have high quality interactions with health service providers

Many barriers to quality interactions were identified, including: local culture and gender norms, education, knowledge of health services and health problems, access to good quality information, communication skills of staff, health worker's attitudes and organizational policy on communication with community members.

When there are one or more of these potential barriers to quality interaction with health service providers, the health literacy of a community member will need to be high.

3. Health literacy is required to optimize caring for one's own health and the health of others

The identified determinants of this area included gender roles and women's autonomy, spousal support along with knowledge and education.

Improving health literacy increases understanding of health and disease as well as the available services, hence people are able to take decisions to take care of their own self and others.

4. Health literacy is required to enable participation in health negotiations and decision-making

The review identified few determinants of participation including the ability to engage in discussions related to gender roles and discrimination, women involvement in decision making, men's involvement in women's health, women's autonomy, spousal support, knowledge and education and the health system responsiveness including communication skills of staff and the quality of health services. Another relevant ability in the community level is the ability of an individual to be able to discuss health matters and make decisions about health. This requires adequate health literacy in an individual and across a community. A strong background mechanism is likely to be educational attainment, including having an understanding of basic biomedical concepts including anatomy and basic medical terms. Without these, being empowered to participate in health negotiations and decision making is unlikely.

which health services can accommodate this diversity, including how services ensure all eligible individuals gain equitable access to the services they provide. Improved health literacy can enhance doctor-patient communication by patients making more informed choices and doctors communicating in plain language to increase the patients' understanding of their health [72]. Health literacy responsive healthcare professionals can also contribute to improving health literacy of patients by responding to the patients based on their health literacy levels. 
At the community level, health literacy has many implications regarding daily decisions about health promotion and disease management, not only at the individual level, but decisions for and by family and community units. Health literacy in this setting also has profound implications for an individual's and community's ability to comprehend and engaging in negotiations and decision making about health [9].

Knowledge and education are direct determinants of understanding, analysing and critical appraisal abilities which enable people to be aware of the available services and overall understanding of health and disease. Notwithstanding education, the impact of inadequate income, pervasive inequitable cultural practices and poor quality of care, can make decision making about health extremely challenging. While the level of education attained is deemed important [30, 32, 33, 36, 38, 51-54], a lack in knowledge also exists regarding either availability of services, severity of illnesses and/or vulnerability to diseases [48-50, 56-58, 60-62]. Healthcare practices in households have deep roots in cultural beliefs and gender roles [19] thus a strong education system is required to advance this area. Nepal clearly has work to do to strengthen community level health literacy and this will underpin the attainment of SDG3: Ensure healthy lives and promote well-being for all at all ages.

Healthcare engagement barriers include actual and perceived barriers such as income and price as demonstrated that once the services are subsidized or made free, uptake is increased [71]. There is potential for catastrophic health expenditure that can happen at the household level [73]. These costs affect the uptake of services which in turn will affect the attainment of SDGs. Quality of care is determined by the technical expertise, communication skills, attitudes and policy communication at local and regional levels, but are also strongly related to education and cultural beliefs. While quality of care is more a reflection of the healthcare system, the education and cultural beliefs also strongly determine healthcare service utilisation. Beliefs and behavior can change in individuals and communities through effective communication alongside provision of appropriate physical infrastructure, equipment, physical distribution of facilities and availability of staff. These are factors that require well planned capital investment by central and regional government authorities. Table 3 outlines the SDG3 targets and the factors identified that may impact on Nepal's ability to attain the targets. The factors underline the social determinants of health in Nepal at both structural and intermediate levels which are needed to be addressed to attain SDG3. Health literacy interventions have the potential to act on people as well as health system to improve health of the people [2].

\section{Interventions to improve health literacy, health and equity}

While the health sector, including individual health professionals, are major contributors to improving health literacy of the population, the attainment of SDG3 requires collective efforts from all sectors. While health literacy is identified as a "foundation block" for improving global health by the WHO [23] there has been little discussion about how health literacy can be operationalized at scale to achieve such objectives. The South East Asian Regional Office of the WHO recently published a Health Literacy Toolkit for Low- and Middle-Income Countries, which provides insights into how health literacy can be used to impact on systems, services and policy [2, 74]. While our review identified four health literacy intervention points, a process for moving from problem identification to problem solving is required. The toolkit provides guidance for the development and implementation of interventions to address many determinants of health. The toolkit introduced the term "health literacy responsiveness" i.e., "the way in which services, environments and products make health information and support available and accessible to people with different health literacy strengths and limitations" [2]. This concept fits well with the findings of our review. For Nepal to make systematic improvements at scale, locally derived and tailored interventions need to be generated and implemented. A promising approach for undertaking this is outlined in the toolkit, i.e., a health literacy-focused approach to community development called, Ophelia (OPtimising HEalth LIteracy and Access) $[2,75]$. This type of locally derived intervention approach could be coupled with national health literacy needs assessment using health literacy questionnaires specifically designed to guide intervention development.

\section{Strengths and limitations of the study}

The application of the rapid review approach may have led to omission of some published papers. Furthermore, as we did not focus specifically on interventions, the quality assessment could not be applied in detail to generate an overall quality assessment. Nonetheless, this is the first review of a developing country's status in regard to health literacy and its capacity to respond to the SDGs. It forms a reasonable baseline for Nepal and may be a good exemplar for other low and middle income countries to use to scope current status and what is required for health literacy capacity development to impact on SDG3 and other SDGs.

\section{Conclusion}

While Nepal has challenges ahead to attain the SDG3, this rapid review provides some insights to promote discussion and planning in support of an effective plan. In a resource-challenged country facing 
Table 3 SDG3 - ensure healthy lives and promote well-being for all at all ages: targets and factors likely to influence attainment of health-related goals

Health related of SDG 3: ensure healthy lives and promote well-being for all at Factors influencing the attainment of targets
all ages

3.1 By 2030 , reduce the global maternal mortality ratio to less than 70 per 100,000 live births

3.2 By 2030, end preventable deaths of newborns and children under 5 years of age, with all countries aiming to reduce neonatal mortality to at least as low as 12 per 1000 live births and under-5 mortality to at least as low as 25 per 1,000 live births

3.3 By 2030, end the epidemics of AIDS, tuberculosis, malaria and neglected tropical diseases and combat hepatitis, water-borne diseases and other communicable diseases

3.4 By 2030, reduce by one third premature mortality from non-communicable diseases through prevention and treatment and promote mental health and well-being

3.5 Strengthen the prevention and treatment of substance abuse, including narcotic drug abuse and harmful use of alcohol

3.6 By 2020, halve the number of global deaths and injuries from road traffic accidents

3.7 By 2030, ensure universal access to sexual and reproductive health-care services, including for family planning, information and education, and the integration of reproductive health into national strategies and programmes

3.8 Achieve universal health coverage, including financial risk protection, access to quality essential health-care services and access to safe, effective, quality and affordable essential medicines and vaccines for all

3.9 By 2030, substantially reduce the number of deaths and illnesses from hazardous chemicals and air, water and soil pollution and contamination

3.a Strengthen the implementation of the World Health Organization Framework Convention on Tobacco Control in all countries, as appropriate

3.b Support the research and development of vaccines and medicines for the communicable and non-communicable Diseases that primarily affect developing countries, provide access to affordable essential medicines and vaccines, in accordance with the Doha Declaration on the TRIPS Agreement and Public Health, which affirms the right of developing countries to use to the full extent the provisions in the Agreement on Trade-Related Aspects of Intellectual Property Rights (TRIPS) regarding flexibility to protect public health, and in particular provide access to medicines for all

3.c Substantially increase health financing and the recruitment, development, training, and retention of the health workforce in developing countries, especially in the least-developed countries and developing small island states

3.d Strengthen the capacity of all countries, in particular developing countries, for early warning, risk reduction, and management of national and global health risks

Income and Cost

- Cost of Services

- Cost of Transport

- Income status

- Employment status

- Socioeconomic status

Culture and gender

- Gender roles/discrimination

- Cultural norms of women involvement in decision making

- Men's involvement in women's health

- Womens' autonomy

- Spousal support

Knowledge and Education

- Education status

- Knowledge of services

- Knowledge of health problems

- Knowledge of hazards

- Knowledge of Economic burden

- Access to good quality information

Quality of services

- Health system responsiveness

- Infrastructure

- Availability of Services

- Communication skills of staff

- Health worker's attitude

- Human resources for health

- Technical/Managerial competence of staff

- Policy and its implementation

- Policy communication with people

- Privacy/Confidentiality

- Satisfaction regarding healthcare substantial burden of disease, health programs in Nepal are often in competition with other personal, family, community and national priorities where trade-offs need to be made between caring for health and attending to other pressing concerns. Knowledge, awareness, culture, language and communication are among the major barriers for health in Nepal where a comprehensive health literacy approach has potential to contribute in improving the health system. While further research on health literacy is clearly needed, there is an immediate role for health literacy in supporting timely utilisation of health services, strengthening health systems; improving health outcomes and reducing health inequities in Nepal. 


\section{Acknowledgements}

We would like to thank Dr Sarity Dodson for providing input during the conception of this paper.

\section{Funding}

No funding was available for this study. Richard Osborne was funded in part through an Australian National Health and Medical Research Council (NHMRC) Senior Research Fellowship \#APP1059122.

\section{Availability of data and materials}

Data sharing is not applicable to this article as no datasets were generated or analysed during the current study.

\section{Authors' contributions}

SSB contributed to the conception of the review, literature search, study design and writing and revision of various drafts. PKP contributed to the conception and reviewed various drafts. SG contributed to the conception, design and writing the revised draft of the review. SL contributed to conception, literature search and writing of the draft. MB contributed to the literature search, design and edited various drafts. RHO contributed to design, reviewed and edited various drafts. All authors approved the final manuscript to be submitted for publication.

\section{Competing interests}

The authors declare that they have no competing interests.

\section{Consent for publication}

Not applicable.

\section{Ethics approval and consent to participate} Not applicable.

\section{Publisher's Note}

Springer Nature remains neutral with regard to jurisdictional claims in published maps and institutional affiliations.

\section{Author details}

${ }^{1}$ School of Public Health \& Community Medicine, B P Koirala Institute of Health Sciences, Dharan 56700, Nepal. World Health Organization, South-East Asia Regional Office, New Delhi, India. ${ }^{3}$ Health Systems Improvement Unit, Centre for Population Health Research, Faculty of Health, Deakin University, Burwood, Australia.

Received: 22 August 2016 Accepted: 23 March 2017

Published online: 27 March 2017

\section{References}

1. World Health Organization. Health Promotion Glossary. Health Promot Int. 1998;13(4):349-64.

2. Dodson S, Good S, Osborne RH. Health literacy toolkit for low and middleincome countries: a series of information sheets to empower communities and strengthen health systems. New Delhi: World Health Organisation, Regional Office for South East Asia; 2015.

3. DeWalt DA, Berkman ND, Sheridan S, Lohr KN, Pignone NP. Literacy and health outcomes: a systematic review of the literature. J Gen Intern Med 2004;19:1228-39.

4. Al Sayah F, Majumdar SR, Williams B, Robertson S, Johnson JA. Health literacy and health outcomes in diabetes: a systematic review. J Gen Intern Med. 2013;28:444-52.

5. Bostock S, Steptoe A. Association between low functional health literacy and mortality in older adults: longitudinal cohort study. BMJ. 2012;344:e1602.

6. Aboumatar HJ, Carson KA, Beach MC, Roter DL, Cooper LA. The impact of health literacy on desire for participation in healthcare, medical visit communication, and patient reported outcomes among patients with hypertension. J Gen Intern Med. 2013;28:1469-76.

7. Buchbinder R, Butterham R, Ciciriello S, Newmann S, Horgan B, Ueffing E, et al. Health literacy: what is it and why is it important to measure? J Rheumatol. 2011;38(8):1791-7.

8. Jordan JE, Buchbinder R, Osborne RH. Conceptualising health literacy from the patient perspective. Patient Educ Couns. 2010;79(1):36-42.
9. Batterham RW, Hawkins M, Collins PA, Buchbinder R, Osborne RH. Health literacy: applying current concepts to improve health services and reduce health inequalities. Public Health. 2016;132:3-12.

10. Paasche-orlow MK, Wolf MS. Promoting Health Literacy Research to Reduce Health Disparities. J Health Commun. 2010;15:34-41.

11. United Nations Development Programme. Human Development Report 2014. New York: United Nations Development Programme; 2014.

12. World Health Organisation. Federal Democratic Republic of Nepal, Country Cooperation Strategy at a glance. Geneva: World Health Organisation; 2014.

13. Central Bureau of Statistics. National Population and Housing Census 2011. Vol. 1. Kathmandu: Central Bureau of Statistics; 2012.

14. Ministry of Health and Population (MOHP) Nepal. Nepal Demographic and Health Survey 2011. Kathmandu: Ministry of Health and Population (MOHP) Nepal; 2012.

15. Asian Development Bank. Basic 2016 Statistics. Manila: Asian Development Bank; 2016.

16. Stroermer M, Fuerst F, Rijal K, Bhandari R, Nogier C, Gautam GS, et al. In: Sellars-Shrestha S, editor. Review of Community-based Health Insurance Initiatives in Nepal. Kathmandu: giz Deutsche Gesellschaft fur Internationale Zusammenarbeit (GIZ) GmbH; 2012

17. Karki A. HRH challenges and role of private sector: Nepal's experiences. Bangkok: Prince Mahidol Award Conference; 2014.

18. Bhandari GP, Angdembe MR, Dhimal M, Neupane S, Bhusal C. State of non-communicable diseases in Nepal. BMC Public Health. 2014;14:23.

19. Budhathoki SS, Bhattachan M, Yadav AK, Upadhyaya P, Pokharel PK. Eco-social and behavioural determinants of diarrhoea in under five children of Nepal: A framework analysis of the existing literature. Trop Med Health. 2016;44:7.

20. Shrestha MK, Guo CW, Maharjan N, Gurung R, Ruit S. Health literacy of common ocular diseases in Nepal. BMC Opthalmology. 2014;14:2.

21. Vaidya A, Karki DB, Krettek A. Cardiovascular Health Literacy in Nepal: Increasing awareness of cardiovascular diseases is important but inadequate. J Gen Pract Emerg Med Nepal. 2011;2(3):5-7.

22. Shrestha A, Singh SB, Maskey R, Khanal VK, Bhattarai S, Pokharel PK. Health Literacy and its Association with Knowledge of Chronic Diseases in Eastern Nepal. In: Institute for Healthcare Advancement's 15th Annual Health Literacy Conference. California: Institute for Healthcare Advancement; 2016.

23. World Health Organization. United Nations Development Program. Policy brief 4 : Health literacy. In: 9th Global Conference on Health Promotion. Geneva: World Health Organization; 2016. p. 1-9.

24. Khangura S, Konnyu K, Cushman R, Grimshaw J, Moher D. Evidence summaries: the evolution of a rapid review approach. Syst Rev. 2012;1(1):10.

25. Hotchkiss DR. Expansion of rural health care and the use of maternal services in Nepal. Health Place. 2001;7:39-45.

26. O'Donnell O. Access to Health Care in Developing Countries: Breaking Down Demand Side Barriers. Cad Saúde Pública. 2007;23(12):2820-34.

27. Cochrane. The GRADE approach. In: Higgins JP, Green S, editors. Cochrane Handbook for Systematic Reviews of Interventions. 5th ed. The Cochrane Collaboration. 2011. p. 361-2.

28. Shea BJ, Grimshaw JM, Wells GA, Boers M, Andersson N, Hamel C, et al. Development of AMSTAR: a measurement tool to assess the methodological quality of systematic reviews. BMC Med Res Methodol. 2007;7:10.

29. Mishra P, Hansen EH, Sabroe S, Kafle KK. Socio-economic status and adherence to tuberculosis treatment: a case-control study in a district of Nepal. Int J Tuberc Lung Dis. 2005:9(10):1134-9.

30. Bhatta DN, Aryal UR. Paternal Factors and Inequity Associated with Access to Maternal Health Care Service Utilization in Nepal: A Community Based Cross-Sectional Study. PLoS ONE. 2015;10(6), e0130380.

31. Pokhrel S, Sauerborn R. Household decision-making on child health care in developing countries: the case of Nepal. Health Policy Plan. 2004;19(4):218-33.

32. Sharma SK, Sawangdee $Y$, Sirirassamee B. Access to Health: Women's status and Utilization of Maternal Health Services in Nepal. J Biosoc Sci. 2007:39(5):671-92.

33. Baral YR, Lyons K, Skinner J, Van Teijlingen ER. Maternal health services utilisation in Nepal: Progress in the new millennium? Heal Sci J. 2012;6(4):618-33.

34. Pokhrel S. Determinants of parental reports of children's illnesses: Empirical evidence from Nepal. Soc Sci Med. 2007;65(6):1106-17.

35. Ministry of Health and Population [Nepal]. New Era, ICF International. Kathmandu: Nepal Demographic Health Survey; 2011. 
36. Furuta BM, Salway S. Women's Position within the Household as a Determinant Of Maternal Health Care Use in Nepal. Int Fam Plan Perspect. 2006;32(1):17-27.

37. Allendorf K. Couples' reports of women's autonomy and health-care use in Nepal. Stud Fam Plann. 2007;38(1):35-46.

38. Shah R, Rehfuess EA, Maskey MK, Fischer R, Bhandari PB, Delius M Factors affecting institutional delivery in rural Chitwan district of Nepal: a community-based cross-sectional study. BMC Pregnancy Childbirth. 2015;15:27.

39. Chapagain M. Conjugal Power Relations and Couples' Participation in Reproductive Health Decision-Making: Exploring the Links in Nepal. Gend Technol Dev. 2006;10(2):159-89.

40. Mullany BC, Becker S, Hindin MJ. The impact of including husbands in antenatal health education services on maternal health practices in urban Nepal: results from a randomized controlled trial. Health Educ Res. 2007;22(2):166-76.

41. Atteraya MS, Kimm H, Song $\mathbb{H}$. Women's autonomy in negotiating safer sex to prevent HIV: Findings from the 2011 Nepal Demographic and Health Survey. AIDS Educ Prev. 2014;26(1):1-12.

42. Allendorf K. Do Women's Land Rights Promote Empowerment and Child Health in Nepal? World Dev. 2007;35(11):1975-88.

43. Upadhyay $P$, Liabsuetrakul $T$, Shrestha $A B$, Pradhan N. Influence of family members on utilization of maternal health care services among teen and adult pregnant women in Kathmandu, Nepal: a cross sectional study. Reprod Biomed Online. 2014;11(1):1-11.

44. Mullany BC. Barriers to and attitudes towards promoting husbands' involvement in maternal health in Katmandu. Nepal Soc Sci Med. 2006;62(11):2798-809.

45. Mullany BC, Hindin MJ, Becker S. Can women's autonomy impede male involvement in pregnancy health in Katmandu, Nepal? Soc Sci Med. 2005;61(9):1993-2006.

46. Smith-estelle A, Gruskin S. Vulnerability to HIV/STIs among Rural Women from Migrant Communities in Nepal: A Health and Human Rights Framework. Reprod Health Matters. 2003;11(22):142-51.

47. Bhattarai S, Parajuli SB, Rayamajhi RB, Paudel IS. Health Seeking Behavior and Utilization of Health Care Services in the Village Development Committees of Ilam District of Nepal. J Chitwan Med Coll. 2015;11(2):8-16.

48. Bhusal CL, Singh SP, BC RK, Dhimal M, Jha BK, Acharya L, et al. Effectiveness and efficiency of Aama Surakshya Karyakram in terms of barriers in accessing maternal health services in Nepal. J Nepal Health Res Counc. 2011;9(2):129-37.

49. Powell-Jackson T, Morrison J, Tiwari S, Neupane BD, Costello AM. The experiences of districts in implementing a national incentive programme to promote safe delivery in Nepal. BMC Health Serv Res. 2009;9:97.

50. Poudel AN, Newlands D, Simkhada P. Economic Burden of HIV/AIDS upon Households in Nepal: A Critical Review. Nepal J Epidemiol. 2015;5(3):502-10.

51. Halim N, Bohara AK, Ruan X. Healthy mothers, healthy children: does maternal demand for antenatal care matter for child health in Nepal? Health Policy Plan. 2011;26:242-56.

52. Acharya DR, Bell JS, Simkhada P, Teijlingen ER V, Regmi PR. Women's autonomy in household decision-making: a demographic study in Nepal. Reprod Health. 2010;7:15

53. Simkhada B, van Teijlingen ER, Porter M, Simkhada P. Major problems and key issues in Maternal Health in Nepal. Kathmandu Univ Med J. 2006;4(14):258-63.

54. Gubhaju B. The Influence of Wives $\hat{a} €^{\mathrm{TM}}$ and Husbands $\hat{a} €^{\mathrm{TM}}$ Education Levels On Contraceptive Method Choice in Nepal, 1996-2006. Int Perspect Sex Reprod Health. 2009;35(4):176-85.

55. Brunson J. Confronting maternal mortality, controlling birth in Nepal: The gendered politics of receiving biomedical care at birth. Soc Sci Med. 2010;71(10):1719-27.

56. Choulagai B, Onta S, Subedi N, Mehata S, Bhandari GP, Poudyal A, et al. Barriers to using skilled birth attendants' services in mid- and far-western Nepal: a cross-sectional study. BMC Int Heal Hum Rights. 2013;13:49.

57. Onta S, Choulagai B, Shrestha B. Perceptions of users and providers on barriers to utilizing skilled birth care in mid- and far-western Nepal: a qualitative study. Glob Health Action. 2014;7:24580.

58. Regmi K, Naidoo J, Greer A, Pilkington P. Understanding the effect of decentralisation on health services: the Nepalese experience. J Health Organ Manag. 2010;24(4):361-82.
59. Iriyama S, Nakahara S, Jimba M, Ichikawa M, Wakai S. AIDS health beliefs and intention for sexual abstinence among male adolescent students in Kathmandu, Nepal: A test of perceived severity and susceptibility. Public Health. 2007;121:64-72.

60. Budhathoki SS, Singh SB, Sagtani RA, Niraula SR, Pokharel PK. Awareness of occupational hazards and use of safety measures among welders: a cross-sectional study from eastern Nepal. BMJ Open. 2014;4(6):e004646.

61. Puri $M$, Cleland J. Sexual behavior and perceived risk of HIV/AIDS among young migrant factory workers in Nepal. J Adolesc Heal. 2006;38:237-46.

62. Poudel KC, Jimba M, Okumora J, Joshi AB, Wakai S. Migrants' risky sexual behaviours in India and at home in far western Nepal. Trop Med Int Heal. 2004;9(8):897-903.

63. Baral YR, Lyons K, Skinner J, van Teijlingen ER. Determinants of skilled birth attendants for delivery in Nepal. Kathmandu Univ Med J. 2010;8(3):325-32.

64. Ghimire L, van Teijlingen ER. Barriers to Utilisation of Sexual Health Services by Female Sex Workers in Nepal. Glob J Health Sci. 2009;1(1):12-22.

65. Byrne A, Hodge A, Jimenez-soto E, Morgan A. Looking Beyond Supply: A Systematic Literature Review of Demand-Side Barriers to Health Service Utilization in the Mountains of Nepal. Asia Pacific J Public Heal. 2013;25(6):438-51.

66. Jahn A, lang MD, Shah U, Diesfeld HJ. Maternity care in rural Nepal: a health service analysis. Trop Med Int Heal. 2000;5(9):657-65.

67. Dhillon PK, Jeemon P, Arora NK, Mathur P, Maskey M, Sukirna RD, et al. Status of epidemiology in the WHO South-East Asia region: Burden of disease, determinants of health and epidemiological research, workforce and training capacity. Int J Epidemiol. 2012;41(3):847-60.

68. De Andrade LOM, Filho AP, Solar O, Rígoli F, De Salazar LM, Serrate PCF, et al. Social determinants of health, universal health coverage, and sustainable development: Case studies from Latin American countries. Lancet. 2015; 385(9975):1343-51.

69. Taggart J, Williams A, Dennis S, Newall A, Shortus T, Zwar N, et al. A systematic review of interventions in primary care to improve health literacy for chronic disease behavioral risk factors. BMC Fam Pract. 2012;13:49.

70. Sheridan SL, Halpern DJ, Viera AJ, Berkman ND, Donahue KE, Crotty K. Interventions for Individuals with Low Health Literacy: A Systematic Review. J Health Commun. 2011;16(3):30-54.

71. Witter S, Khadka S, Nath H, Tiwari S. The national free delivery policy in Nepal: Early evidence of its effects on health facilities. Health Policy Plan. 2011;26(2):ii84-91.

72. Beauchamp A, Buchbinder R, Dodson S, Batterham RW, Elsworth GR, McPhee $C$, et al. Distribution of health literacy strengths and weaknesses across socio-demographic groups: a cross-sectional survey using the Health Literacy Questionnaire (HLQ). BMC Public Health. 2015;15(1):678.

73. Saito E, Gilmour S, Rahman MM, Gautam S, Shrestha K, Shibuya K Catastrophic household expenditure on health in Nepal : a cross- sectional survey. Bull World Heal Organ. 2014;92:760-7.

74. Budhathoki SS. WHO-SEARO Toolkit: a call for action in Nepal to improve the health of the Nepalese Population [Internet]. BMJ. 2015 [cited 2016 Mar 2]. Available from: http://www.bmj.com/content/350/bmj.h1026/rr-0.

75. Batterham RW, Buchbinder R, Beauchamp A, Dodson S, Elsworth GR, Osborne RH. The OPtimising HEalth LIterAcy (Ophelia) process: study protocol for using health literacy profiling and community engagement to create and implement health reform. BMC Public Health. 2014;14(1):694.

76. Regmi PR, van Teijlingen E, Simkhada P, Acharya DR. Barriers to Sexual Health Services for Young People in Nepal. J Heal Popul Nutr. 2010;28(6):619-27.

\section{Submit your next manuscript to BioMed Central and we will help you at every step:}

- We accept pre-submission inquiries

- Our selector tool helps you to find the most relevant journal

- We provide round the clock customer support

- Convenient online submission

- Thorough peer review

- Inclusion in PubMed and all major indexing services

- Maximum visibility for your research

Submit your manuscript at www.biomedcentral.com/submit 